\title{
Intermédialités
}

Histoire et théorie des arts, des lettres et des techniques

Intermediality

History and Theory of the Arts, Literature and Technologies

\section{Psycho/Bates Motel : hyperdiégèse et réactivation sélective}

\section{Marta Boni}

Numéro 28-29, automne 2016, printemps 2017

refaire

redoing

URI : https://id.erudit.org/iderudit/1041077ar

DOI : https://doi.org/10.7202/1041077ar

Aller au sommaire du numéro

Éditeur(s)

Revue intermédialités (Presses de l’Université de Montréal)

ISSN

1920-3136 (numérique)

Découvrir la revue

Citer cet article

Boni, M. (2016). Psycho/Bates Motel : hyperdiégèse et réactivation sélective. Intermédialités / Intermediality, (28-29). https://doi.org/10.7202/1041077ar
Résumé de l'article

Cette contribution propose un modèle pour l'analyse de la tendance contemporaine consistant à refaire, en série, un film culte. L'analyse de la série Bates Motel (A\&E, 2013-), choisie comme exemple pertinent, s'appuie sur l'insertion de la série dans une sphère de discours plus large représentée par l'univers de Psycho et ses multiples incarnations médiatiques, incluant les appropriations « apocryphes » des téléspectateurs. Bates Motel correspond ainsi à l'exploration d'un espace. Ce phénomène est décrit à travers les concepts d'hyperdiégèse de Matt Hills (Fan Cultures, Londres, New York, Routledge, 2002), ainsi que de série culturelle et de musée imaginaire de Martin Lefebvre (Psycho : de la figure au musée imaginaire. Théorie et pratique de l'acte de spectature, Montréal, Paris, L’Harmattan, 1997). Les notions d'« activation » ou de « réactivation sélective » sont par ailleurs proposées et discutées. 


\title{
Psycho/Bates Motel : hyperdiégèse et réactivation sélective
}

\author{
MARTA BONI
}

Chick: So the best version [...] is not for me to just recreate somebody else's work. It's for me to be inspired by that work and then create something else that has its own life and purpose and form of integrity, and reason for being.

omme le montre la prolifération d'adaptations, sequels, prequels et reboots cinématographiques de séries télévisées, notable dans les années 1990 (Twin Peaks. Fire Walk With Me, Lynch, 1991; The X Files. Fight the Future, Bowman, 1998 et The X Files: I Want to Believe, Carter, 2008), et, plus récemment, la création de séries à partir d'un film culte (Fargo, FX, 2015-; Scream, MTV, 2015-), la production audiovisuelle est plus que jamais marquée par une tendance à refaire à partir de matériel existant. Dans le panorama américain des trois dernières années, il est possible de compter environ quarante-cinq séries télévisées qui adaptent des films ${ }^{2}$. Ces bouillonnements intermédiaux ne relèvent pas d'un phénomène nouveau : des exemples de ce type de circulation ont toujours caractérisé les relations entre le cinéma et la télévision, à partir de la période de l'institutionnalisation de celleci, dans les années $1950^{3}$. Dans cet article, nous interrogerons les effets de la remise en

I Bates Motel, 2013-, Anthony Cipriano, Carlton Cuse et Kerry Ehrin, saison 4, épisode 5 : « Refraction », diffusé le ir avril 20ı6, New York, A\&E.

${ }^{2}$ Sources mixtes: Wikipédia, Senscritique, Rotten Tomatoes, IMdB.

3 Ina Rae Hark, «The Wrath of the Original Cast. Translating embodied television characters to other media », dans Deborah Cartmell et Imelda Whelehan (dir.), Adaptations: From Text to Screen, Screen to Text, Londres, New York, Routledge, 1999, p. 172-184. Voir aussi Shannon Wells-Lassagne, Television and Serial Adaptation, New York, Londres, Routledge, 2017. 
circulation de certaines portions de mondes diégétiques ou de formes esthétiques, dans la perspective d'une réactivation sélective.

Si la sérialité, définie comme une alternance de répétition et d'innovation, est une constante dans l'histoire de la production artistique mondiale — à l'exception de la modernité, qui érigeait l'unique et l'original en valeurs de création ${ }^{4}$-, aujourd'hui, la sérialisation d'un contenu qui à l'origine a été pensé pour être une unité discrète devient la norme. L'étendue des stratégies de reprise de type sériel comme choix industriel est d'autant plus visible dans un moment historique marqué par l'émergence de grands conglomérats médiatiques et d'un marché qui voit primer l'image de marque et la franchise ${ }^{5}$. Dans ce contexte, on remarque une « abondance ${ }^{6}$ » de contenus télévisuels, la présence d'un choix presque illimité de programmes 7 et, sur le plan des formes, une plus grande complexité, autoréférentialité et intertextualité ${ }^{8}$. Le cinéma, par ailleurs, est aussi pris dans une logique sérielle, comme le montre la remarque suivante :

The problem for Hollywood is that audiences are ignoring everything that isn't a sequel, adaptation, or reboot. The market for films based on stories that aren't already famous is threadbare. These sort of stories exist in entertainment, but consumers, and particularly young consumers, are looking for them outside of darkened theaters?.

Tout en mettant en évidence l'élasticité des frontières entre les médias qui est inscrite dans l'acte de refaire une œuvre, ces phénomènes entraînent la dilatation transfictionnelle d'un contenu, d'un personnage ou d'un monde imaginaire ${ }^{\mathrm{IO}}$. Les personnages s'autonomisent du texte et vivent plus aisément que d'habitude des mutations de leur sort, par exemple, ou changent de période historique et même de sexe, comme le docteur Watson qui, dans la série Elementary (CBS, 2012-), est

4 Umberto Eco, «Innovation and Repetition: Between Modern and Post-Modern Aesthetics », Daedalus, vol. II4, $\mathrm{n}^{\circ}$ 4, automne I985, p. I6I-I84.

5 Derek Johnson, Media Franchising: Creative License and Collaboration in the Culture Industries, New York, New York University Press, 2013.

${ }^{6}$ John Ellis, Seeing Things: Television in the Age of Uncertainty, Londres, IBTauris, 2000.

7 Amanda D. Lotz, The Television Will Be Revolutionized, New York, New York University Press, 2007.

${ }^{8}$ Jason Mittell, Complex TV: The Poetics of Contemporary Television Storytelling, New York, New York University Press, 2015.

9 Derek Thompson, « Hollywood Has a Huge Millennial Problem », The Atlantic, 8 juin 2016.

Io Richard Saint-Gelais, Fictions transfuges: la transfictionnalité et ses enjeux, Paris, Éditions du Seuil, coll. « Poétique », 2011. 
interprété par l'actrice Lucy Liu. Dans ce cas, le fidèle associé de Sherlock Holmes est, tout comme son partenaire, un signifiant flottant: un contenant, reconnaissable à sa seule silhouette, qui maintient son identité malgré ses métamorphoses successives ${ }^{\mathrm{II}}$.

Il s'agit de situations dans lesquelles le référent n'est plus le texte mais la diégèse, entendue comme un potentiel, comparable à un iceberg dont seule la pointe est visible, qui cache un matériel bien plus large. Matt Hills parle d'hyperdiégèse, caractéristique du texte culte, qu'il décrit comme «la création d'un espace narratif large et détaillé, dont on ne voit ou rencontre directement qu'une portion dans le cadre du texte, mais qui toutefois semble fonctionner selon des principes de logique interne et d'expansion ${ }^{12} \gg$. Rappelons que, selon Eco, tout texte est une machine $\dot{a}$ produire des mondes possibles ${ }^{13}$.

Refaire une ouvre n'est pas exclusivement la prérogative d'un adaptateur officiel. Au contraire, chaque expérience spectatorielle correspond à l'acte de sélectionner des éléments prégnants pour les inclure dans une lecture personnelle qui est aussi, souvent, une réécriture. Toute réception engendre dès lors, avec plusieurs nuances, un acte de refaire. On peut lire ce concept dans le véritable sens d'un art de faire certaldien : les spectateurs, en refaisant une ouvre, se l'approprient, l'habitent, lui attribuent des significations identitaires ${ }^{14}$. Les traces de ces appropriations deviennent de nouveaux points de départ pour une circulation d'interprétations dans l'espace social (remarquons que ces traces sont d'autant plus visibles dans le contexte de la culture participative d'Internet). C'est dans ce contexte, dans lequel des termes comme adaptation, remake ou citation paraissent insuffisants, que la perspective du reenactment devient particulièrement productive. Le reenactment sera ici conçu comme acte performatif de mémoire et de construction d'un accrochage inédit,

${ }^{\text {II }}$ Roberta E. Pearson et William Uricchio (dir.), The Many Lives of the Batman: Critical Approaches to a Superhero and his Media, New York, Londres, Routledge, BFI Publishing, I99I.

${ }_{12}$ « Another defining attribute of the cult text is hyperdiegesis: the creation of a vast and detailed narrative space, only a fraction of which is ever directly seen or encountered within the text, but which nevertheless appears to operate according to principles of internal logic and extension. » Matt Hills, Fan Cultures, Londres, New York, Routledge, coll. « Sussex studies in culture and communication », 2002, p. 104 (notre traduction).

${ }^{13}$ « But the text is not a possible world-nor is the plot. It is a piece of the furniture of the world in which the reader also lives, and it is a machine for producing possible worlds (of the fabula, of the characters within the tabula, and of the reader outside the fabula) », Umberto Eco, The Role of the Reader: Explorations in the Semiotics of Texts, Bloomington, Indiana University Press, coll. « Midland books; Advances in semiotics », 1984, p. 246.

${ }^{14}$ Michel de Certeau, Arts de faire, Paris, Union générale d'éditions, coll. « Le monde en Io/I8; Invention du quotidien », 1980 . 
comme on le verra à travers l'image du musée imaginaire, dans un nouveau contexte, d'éléments provenant d'un texte ou d'un ensemble de textes.

Penser les phénomènes d'adaptation d'un film en série demande donc d'en mettre en évidence les usages culturels ainsi que leurs effets réciproques. En plus de la sérialisation industrielle, il est nécessaire de prendre en compte, comme autant de composantes d'une sphère de discours plus large et en évolution constante dans le temps, les réécritures «apocryphes » et les autres traces des appropriations des téléspectateurs et des fans, comme les fan fictions, nombreuses dans les espaces en ligne d'Internet. Une recherche dans les territoires de la discursivité grassroots, effectuée entre mai et septembre 2016, nous permet de laisser émerger des caractéristiques de ces pratiques de remise en circulation de portions de mondes, qui prennent vie pendant le développement d'une série, elle-même reenactment d'un monde complexe. Nous rendrons compte de la variété des pratiques par une analyse de type qualitatif de quelques exemples significatifs choisis parmi un large corpus de traces de discours issues du territoire du Web. Dans leur ensemble, ces appropriations vont entourer le texte d'origine, brouillant les hiérarchies, produisant une superposition sémiotique dans le temps. Lorsque nous analyserons leurs détails, nous pourrons souligner certains effets de ce phénomène et notamment le fait que, au sein de la sphère de discours, des activations sélectives de certains éléments deviennent plus visibles à des moments déterminés. Par activation sélective on entendra le phénomène d'apparition, ou de surbrillance, de certaines composantes, choisies par l'interprétant collectif comme signifiants au sein d'un processus complexe ${ }^{\mathrm{IS}}$, non limité à la série télévisuelle mais étendu à la série culturelle ${ }^{16}$ représentée par les différentes incarnations médiatiques d'une hyperdiégèse.

Bates Motel (A\&E, 2013-) ${ }^{17}$ confirme la pertinence des remarques sur ce paradigme de sérialité complexe. La série fait partie d'un monde, étant à la fois l'espace diégétique dans lequel se déroule le récit, pensé selon les codes de la complexité narrative contemporaine, et espace de discours plus large, la série culturelle issue de Psycho. Cette dernière englobe des appropriations variées qui garantissent son

is Le phénomène est comparable aux « émergences » dans la théorie des systèmes complexes.

${ }^{16}$ Martin Lefebvre, Psycho: de la figure au musée imaginaire. Théorie et pratique de l'acte de spectature, Montréal, Paris, L'Harmattan, coll. « Champs visuels », 1997.

${ }^{17} \mathrm{La}$ série est produite par Roy Lee, célèbre pour avoir adapté pour le marché américain des succès d'horreur étrangers, et créée par une équipe dans laquelle figure Carlton Cuse, ayant travaillé avec Damon Lindelof sur la série culte Lost (ABC, 2004-20IO). Le Network A\&E (Arts \& Entertainment) a entre autres produit l'adaptation américaine de la série française Les Revenants (Canal+, Fabrice Gobert, 2012-) sous le titre The Returned (Carlton Cuse, 2015). 
existence, dans un état d'évolution constante. Du point de vue narratif, il s'agit d'une expansion transfictionnelle d'un monde sur le mode de l'hyperdiégèse; du point de vue sémiotique, ce processus peut être lu comme un «musée imaginaire ${ }^{18}$ » ou comme un assemblage particulier, individuel et collectif, à une époque et dans un contexte donnés, d'éléments signifiants.

\section{DE RETOUR CHEZ LES BATES}

L'émission d'A\&E nous permet de plonger dans l'intimité des Bates, mère et fils, à une période qui se situe avant les événements racontés par Psycho (Hitchcock, 1960) ${ }^{19}$, mais connotée à l'aide de données « environnementales » contemporaines aux spectateurs. On y côtoie un Norman adolescent, âgé de I7 ans, et sa mère, Norma, fuyant tous deux un passé tumultueux (les premières images nous apprennent que le père de Norman est mort dans des circonstances violentes dont Norma semble, au premier abord, être responsable) et tentant de mener une vie acceptable ${ }^{20}$ dans le cadre d'une petite ville du Nord-Ouest Pacifique, White Pine Bay.

Si le texte hitchcockien est indéniablement le point de départ, la série Bates Motel est aussi à considérer comme une des nombreuses composantes d'un système médiatique complexe ${ }^{2 \mathrm{I}}$, avec lesquelles elle affiche de nombreux liens de type intertextuel et hypertextuel. Les différents sequels et prequels de Psycho, ainsi que le remake de Gus Van Sant (Psycho, 1998) et le film pour la télévision de 1987 portant le titre de Bates Motel, convergent tous vers cette version rafraîchie du classique, destinée aux générations de téléspectateurs « de I 8 à 34 ans ». Sur le plan du récit, le lien intertextuel est affiché de manière claire à travers des citations directes et des clins d'œil plus subtils, résultant en la création de ce que Andrew Scahill appelle des prémonitions $^{22}$, qui font appel à la mémoire de certains spectateurs. Poursuivant dans cette direction, on pourrait remarquer que la série joue, en même temps, sur le thème hitchcockien par excellence : le revenant, largement étudié dans le cas de Vertigo

${ }^{18}$ Lefebvre, 1997.

${ }^{19} \mathrm{La}$ série affiche dans son paratexte (le générique de fin) le lien direct avec le film Psycho et le livre de Robert Bloch (1959) avec la formule « librement inspiré de... ».

${ }^{20}$ Récurrence hitchcockienne: dans Marnie (1964), on rappelle l'insistance, dans les discours de la mère, ancienne prostituée préoccupée de la tenue convenable de la protagoniste, sur les termes « decent » et « respectable ».

${ }^{21}$ «Psycho (franchise) », Wikipédia, https://en.wikipedia.org/wiki/Psycho_(franchise) (consultation le is septembre 2016).

${ }^{22}$ Andrew Scahill, « Serialized Killers: Prebooting Horror in Bates Motel and Hannibal », dans Amanda Ann Klein et R. Barton Palmer (dir.), Cycles, Sequels, Spin-Offs, Remakes, and Reboots: Multiplicities in Film and Television, Austin, University of Texas Press, 2016, p. 322. 
(Hitchcock, 1958). Selon la perspective du reenactment, le passé est à considérer comme un espace que l'on peut retravailler, et cela surtout grâce à la dimension en réseau des contenus ainsi qu’à leur disponibilité constante, rendues possibles par le contexte numérique. Grâce aux nouvelles modalités de visionnage et de production qui la délivrent (partiellement) du traditionnel flux et à la grille des programmes, la télévision devient désormais une archive performative : elle conserve et expose des contenus tout en étant un catalyseur d'instances de recréation de sens à partir de ces archives mêmes.

Sur le plan visuel, la série met en branle le partage d'un imaginaire culte : la maison victorienne des Bates, surtout, qui est un incontournable monument mémoriel. On y retrouve certains éléments de la diégèse que développait le film pour la télévision Psycho IV - The Beginning (Perkins, 1990), où Norman adulte (Anthony Perkins), sorti de l'hôpital psychiatrique, marié et dans l'attente d'un bébé, était encore douloureusement hanté par les souvenirs de son adolescence. Par des retours en arrière, le film décryptait le crescendo de la tendance psychotique du personnage, expliquée à travers des épisodes de séduction et d'humiliations violentes subies de la part de sa mère (une terrifiante Olivia Hussey), l'amenant finalement à empoisonner celle-ci et son amant avec de la strychnine (voir la figure I).

Bates Motel s'attache aussi à l'adolescence de Norman. Toutefois, la structure sérielle lui permet de donner davantage d'ampleur aux nuances psychologiques, d'enregistrer les mutations imperceptibles du caractère des protagonistes, ainsi que de développer des récits parallèles. Conformément aux caractéristiques de la sérialité télévisuelle qui, selon Jean-Pierre Esquenazi ${ }^{23}$, transforme les personnages en compagnons des téléspectateurs, la dimension de l'intime y est très présente, l'espace domestique jouant un rôle central.

Suivant le modèle des théories des mondes possibles, on pourrait d'abord se demander : sommes-nous dans le même monde que celui de Psycho ? L'espace se rapproche de celui d'origine, notamment en raison de la maison victorienne et du motel, tout à fait homologues; toutefois, le lieu n'est pas le même. L'action se déroule en effet en Oregon et non plus en Arizona, dans un monde, donc, qu'on pourrait décrire comme parallèle. Le temps est le présent pour le téléspectateur, mais c'est le futur par rapport aux années 1960 de Psycho. Plus précisément, dans le monde réel textuel (textual actual world ${ }^{24}$ ), les personnages n'ont pas de mémoire des événements racontés par le film.

${ }^{23}$ Jean-Pierre Esquenazi, Les séries télévisées : l'avenir du cinéma?, Paris, Armand Colin, coll. « Cinéma », 2 oro.

${ }^{24}$ Marie-Laure Ryan, Possible Worlds, Artificial Intelligence, and Narrative Theory, Bloomington, Indiana University Press, I99I. 


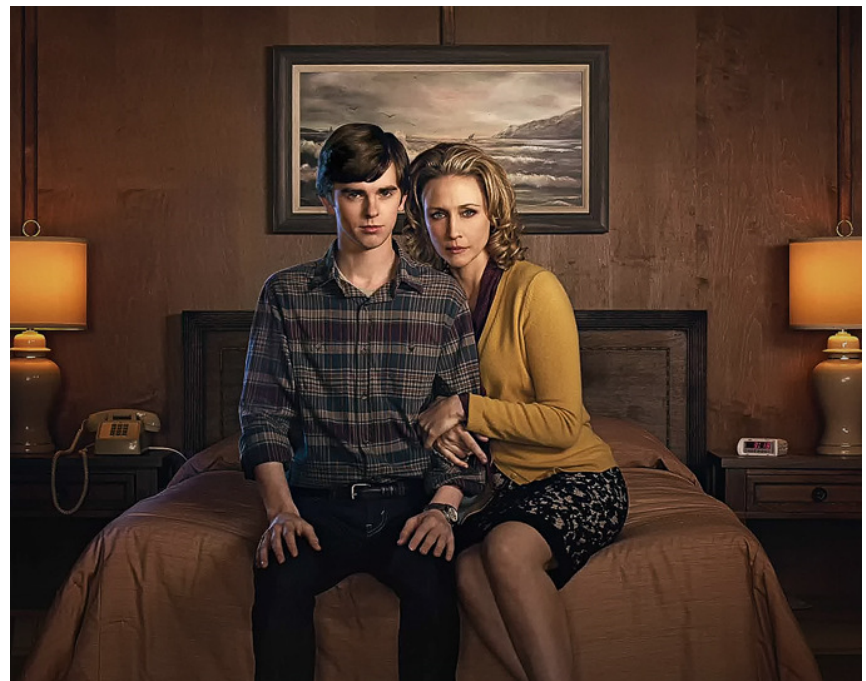

\section{A BOY'S BEST FRIEND IS HIS MOTHER.}
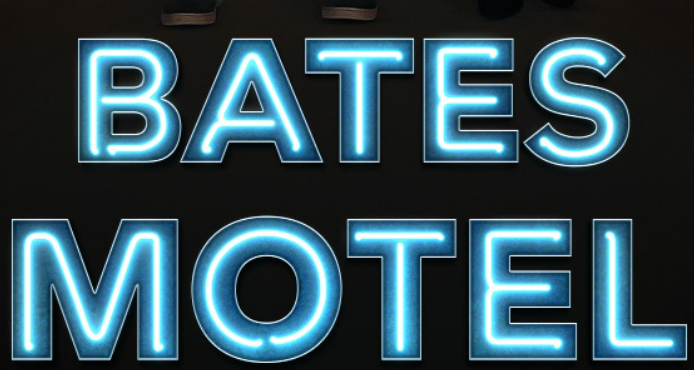

Fig. I. Affiche de la saison I de Bates Motel, 2013.

Cependant, les vêtements de Norman et de Norma, leur voiture ainsi que leur goûts cinématographiques (Double Indemnity, Wilder, 1944) et musicaux ( $M r$. Sandman des Chordettes, 1958) renvoient à un temps suspendu. Conformément à la logique du reboot, la série est un pont entre le monde d'Hitchcock, ou de manière plus large l'époque classique du cinéma (qui est ainsi confirmée comme monde culte, que l'on peut citer), et le monde des nouvelles technologies.

Les téléspectateurs dont l'horizon d'attente se basait sur l'homologie seront déstabilisés. Par exemple, une spectatrice reconnaît :

I have to say I was so excited about this show until the trailer showed Norman listening to Ipod. I'm pretty sure in that short amount of time Alfred 
Hitchcock found a way to roll over in his grave... twice. Being a fan of the movie I'm really disappointed in the direction the show is heading in and all the differences surrounding it [...] The time period really throws me off to [sic], why in the world was this set in present times ${ }^{25}$ ?

Pour d'autres, l'occasion de nouvelles explorations est aussi une façon d'assurer la pérennité de l'original :

$[\ldots]$ it preserves the Hitchcockțs $[$ sic] masterpiece mark innovating and becoming a kind of bridge between different expectations of the viewer... [The story does] not gives [sic] answers but explores new and new possibilities. proposing a coherent world who escapes white/black definitions ${ }^{26}$.

La présence du verbe « préserver » et de l'adjectif « cohérent » signalent, dans ce commentaire, la relation avec un texte de départ. Toutefois, notons qu'il ne s'agit pas d'une relation binaire avec un hypotexte : il s'agit plutôt d'un dispositif similaire à la constitution d'une archive, à l'exploration canonique de toutes les possibilités d'une bible (au sens télévisuel) ou d'un environnement culte. Il serait vain de se concentrer exclusivement sur les éléments de continuité : une approche ethnographique de la réception dans les espaces en ligne nous montre que tous les spectateurs ne connaissent pas le film d'Alfred Hitchcock. Ce qui émerge est l'opération de ratissage d'un territoire ou d'un monde possible qui éclot progressivement au passage de la loupe de l'adaptateur, suivant les besoins de la narration sérielle.

\section{UN RÉCIT SÉRIEL EXPLORATOIRE}

Sérialiser Psycho pour la télévision correspond à « en faire autre chose ». La série multiplie les perspectives, brisant la dimension du rapport de cause à effet. Elle favorise, premièrement, la porosité entre deux lectures génériques, conformément à la mixité typique des narrations complexes de la télévision contemporaine. D’un côté, on retrouve le teen movie, car Norman, adolescent rebelle, évolue dans les couloirs de son lycée (le jeune homme étant vu, dans ce cas, comme l'innocente victime de la folie de sa mère). De l'autre, il s'agit bel et bien d'une série d'horreur, car le jeune homme

${ }^{25}$ Harfordamanda, « Reviews written by registered user Harfordamanda », $I M D b$, I9 mars 2013, http://www.imdb.com/user/ur2090476I/comments (consultation le is septembre 2016).

${ }^{26}$ Kirpianuscus, « Reviews written by registered user Kirpianuscus », $I M D b$, 30 mai 2016, http://www.imdb.com/user/ur6II9753I/comments-expanded?start=67\& order=date (consultation le is septembre 2016). 
semble incarner progressivement le mal absolu, sous l'emprise de son côté « mère » canonique. Le crime, le gangster movie et le soap opera sont également combinés à ces genres primaires ${ }^{27}$.

À l'exploration psychologique s'ajoutent, au cours des épisodes, plusieurs intrigues secondaires, comme les combines de Dylan, demi-frère du protagoniste, qui vont de plus en plus s'ancrer au développement principal (notamment son lien avec des trafiquants de drogue). Elles renforcent le mélange des genres et servent à activer une interprétation ou une autre. La transtextualité, ou la possibilité de penser les personnages et les mondes de manière indépendante du texte, apparaît comme la condition première pour comprendre ces phénomènes de circulation médiatique qui émergent lorsqu'on refait un classique. Loin d'être un calque ou une imitation, l'acte de refaire correspond à la découverte progressive d'un territoire très large.

L'espace de la diégèse se dévoile progressivement. Dans le film d'Hitchcock, tout ce que les spectateurs pouvaient voir du village était la maison du policier et une petite église, alors que les actions principales se déroulaient dans des espaces clos, privant les spectateurs d'une image de l'ensemble. Au contraire, dans Bates Motel, une intrigue concernant les trafiquants de drogue se présente dès le début comme le signe que le petit village de White Pine Bay, à l'instar d'une autre ville télévisuelle, Twin Peaks, contient beaucoup plus que ce que le motel nous annonce dans les guides qu'il met à la disposition de ses clients : sous la surface de la station touristique paisible se cachent des secrets de drogue, de traite d'esclaves sexuelles, de corruption. La question inscrite dans l'acte de refaire Psycho est doncla suivante: comment rendre habitable, comment ré-habiter ce monde?

Nous assistons également à des transformations et à des ajouts qui créent une superposition qui n'efface pas le reste. Loin de détourner l'original, Bates Motel retravaille ce qui de Psycho s'est cristallisé dans le temps au fil des appropriations successives. L'hyperdiégèse nous permet de penser l'existence d'une partie cachée, ou d'une base de données qui demeure dans un état transcendant (non incarné dans une

${ }_{27}$ On peut le voir dans ces commentaires : « I've come to accept that Bates Motel is not a Psycho show. It's this Bizarro universe soap opera that happens to have some characters from the Psycho universe in it. », Drew Dietsch, «TV Review : Bates Motel - Season I Review », The Drew Reviews, 2 juin 2013, http://drewreview.blogspot.ca/2013/06/tv-review-batesmotel-season-I-review.html (consultation le I9 septembre 2016). Ou encore, ils le définissent ainsi : « Wrong and getting wronger - in just the right way »; « its definitely an adult oriented show », Sean Morrow, « Reviews \& Ratings for "Bates Motel” », IMDb, 4 avril 2013, http://www.imdb.com/title/tt218867I/reviews (consultation le is septembre 2016). 
forme médiatique: voir aussi la notion de database narrative ${ }^{28}$ ). Ainsi, le texte originaire, ayant un auteur et une date bien définis, est la pointe de l'iceberg, la plus visible à une époque et dans un contexte donné, alors que les nombreux sequels et prequels, incluant Bates Motel, en sont la portion cachée, mais prête à émerger à chaque nouvelle incarnation médiatique.

La franchise de Psycho est donc un espace de discours, dont le film d'Alfred Hitchcock est le noyau canonisé, reconnaissable, ayant une position précise dans la hiérarchie; d'autres textes, moins connus ou moins célébrés, l'entourent. Suivant le modèle de la sémiosphère de Lotman ${ }^{29}$, nous pouvons considérer ces portions d'univers comme des composantes périphériques d'un système, relatives à un canon et en évolution constante.

\section{MÉMOIRE SÉRIELLE}

Le réseau de relations que supposent la logique hyperdiégétique et la transfiction nous empêche de penser le reboot d'A\&E comme une brique indépendante: Bates Motel fait partie d'un parcours mémoriel composé d'une histoire, d'un monde et de personnages, mais aussi d'une certaine idée du style hitchcockien, qui au fil du temps sont devenus une part du patrimoine collectif de la culture occidentale. La répétition est en cela un acte signifiant, à entendre dans le sens d'une mutation dépendant du renouvellement sémantique qu'impose le nouveau contexte $^{30}$. Elle se constitue par la sélection d'éléments particuliers et par leur assemblage particulier, à lire dans le cadre d'une série culturelle.

Selon Martin Lefebvre, une série est la « façon qu'a un spectateur d'organiser, au sein de sa mémoire et autour d'une figure, un corpus de films ${ }^{31} \gg$. L'expérience de spectature de Psycho génère des figures capables de parcourir de manière transversale les productions successives (remakes, parodies, hommages) et d'autres films qui entretiennent avec l'original des liens plus subjectifs, moins canoniques, comme Carrie (De Palma, 1976), à partir du moment où ils activent une figure chez le

${ }^{28}$ Lev Manovich, «Database as Symbolic Form», Convergence: The International Journal of Research into New Media Technologies, vol. 5, n², juin 1999, p. 80-99.

${ }^{29}$ Yuri M. Lotman, Universe of the Mind: A Semiotic Theory of Culture, Bloomington, Indiana University Press, 1990.

${ }^{30}$ Marie-France Chambat-Houillon, «Entre le même et l'autre : la place de l'auteur », Cinema et cie: International Film Studies Journal, Hans-Michael Bock et Simone Venturini (dir.), $n^{\circ} 6$ « Versions multiples II », printemps 2005 , p. I8.

${ }^{31}$ Lefebvre, 1997, p. 16r. 
spectateur ( « la série regroupe des films qui constituent, en quelque sorte, une famille figurale $32 »)$. La sérialisation résulte de l'agencement ou de l'«accrochage » personnel de plusieurs couches de mémoire, relevant d'expériences différentes, traçant un parcours mémoriel composé de plusieurs textes hétérogènes que Lefebvre définit comme un «musée imaginaire ». Il s'agit d'un rapprochement d'œuvres capable de donner de nouvelles fonctions à celles-ci. Dans le musée moderne de Malraux, isolées du contexte de départ, les œuvres transmettent au visiteur une signification autonome par rapport à leur origine, selon la logique de leur accrochage ${ }^{33}$. Selon Lefebvre, «l'air de famille que reconnaît le spectateur et sur lequel il élabore ici son musée imaginaire ne se limite donc pas à l'identification d'une forme, mais suppose une saisie imaginale commune — d'un film à l'autre - de traits comme le tableau de Suzanne et les vieillards ${ }^{4}$ ». Le musée fonctionne ainsi de manière non hiérarchique et non chronologique: il crée des continuités sur une période élargie, ainsi que des discontinuités et des résultats émergeant de l'assemblage temporaire d'éléments, car « le rapport du texte fondateur aux autres films de la série ne respecte pas forcément l'ordre de parution des films ${ }^{35} \gg$.

C'est dans ce cadre que nous pouvons avancer que la série Bates Motel sélectionne ou active certains traits signifiants, et qu'elle en produit un assemblage inédit ${ }^{36}$. Résultat combinatoire de multiples arts de faire, le musée imaginaire produit ainsi un phénomène d'activation sélective ou, mieux, de réactivation sélective, car il s'agit de composantes qui ont été activées et réactivées plusieurs fois au cours des années. Il est important de souligner que la série culturelle a tout d'abord une importance individuelle pour le spectateur. Toutefois, l'expérience de la spectature est aussi à entendre au sein d'un réseau : il s'agit d'un « ... savoir encyclopédique, un trésor social et culturel qui, même s'il est exploité de façon personnelle, n'en demeure pas moins une propriété commune [...] Subjectivité et solipsisme ne sont pas des

${ }^{32}$ Ibid., p. 162.

33 André Malraux, Les voix du silence, Paris, Gallimard, coll. « NRF Essais », I95I.

${ }^{34}$ Lefebvre, 1997, p. 182.

35 Ibid., p. 168.

${ }^{36}$ Le commentaire suivant d'un internaute souligne l'importance de la série culturelle comme cadre de référence, car il parle à la fois d'Hitchcock et de Psycho $I V$ : « An avid fan of Hitchcock, I thought this pilot was well done. [...] Olivia Hussey left some big shoes to fill after her portrayal of Norma Bates, but I think (given this pilot) Vera Farmiga was well cast by the producers. [...] In order to fully appreciate it, you should watch the 1960 original, and the made-for-TV prequel $\gg$. Kemority, «Reviews \& Ratings for "Bates Motel” », IMDb, 20 mars 2013, http://www.imdb.com/title/tt2I8867I/reviews (consultation le 20 septembre 2016). 
synonymes ${ }^{37} \gg$. La convergence d'expériences individuelles génère, à l'intérieur de l'espace de discours, certaines zones qui seront canonisées de manière collective.

\section{RÉACTIVATION SÉLECTIVE DANS LA SÉRIE TÉLÉVISÉE}

À l'instar du musée imaginaire, Bates Motel propose un accrochage nouveau des éléments fictionnels. Premièrement, on y trouve ce qu'Andrew Scahill nomme des prémonitions. Par exemple, lorsque Norman découvre la taxidermie, l'encyclopédie intertextuelle s'active immédiatement pour ceux qui ont vu Psycho: c'est une invitation à sentir qu'on fait partie d'un club. Il s'agit d'un plaisir rassurant pour les spectateurs. Andrew Scahill va plus loin et affirme que, puisque les spectateurs connaissent déjà l'histoire, le plaisir de Bates Motel se trouve précisément dans le jeu de références connexes, les modes de la réflexivité, l’interaction entre le connu et le nouveau ${ }^{38}$.

Dans ce cadre, le rapport au film matriciel est central en ce qui concerne le genre. Conformément aux traits distinctifs de la télévision complexe ${ }^{39}$, on y retrouve des images particulièrement soignées, une logique non linéaire et la présence de séquences de rêve. Toutefois, si l'horreur hitchcockienne fonctionnait en créant un effet de suspense et de surprise, dans son adaptation elle va se diluer, se perdre (ou devenir camp comme dans le remake de Van Sant). La réécriture sérielle doit donc effectuer un choix, tenant compte de la mémoire générique du spectateur (dont les goûts ont évolué avec les années). Soit on renforcera l'horreur par une logique de surenchère, soit on prendra une autre direction. Bates Motel optera pour cette deuxième possibilité, malgré la persistance, dans certaines séquences, d'un goût slasher contemporain. En l'absence de la musique de Bernard Herrmann qui avait caractérisé Psycho ainsi que ses remakes et ses prequels, le suspense disparaît, remplacé par l'opération (typique du feuilleton) qui consiste à relancer sans cesse l'action après chaque chute. Les meurtres ponctuent ainsi le tissu sériel : la nature du personnage de Norman se dévoile non pas lors d'une révélation centrale, mais à travers de fausses pistes et des superpositions de cas similaires qui vont créer un crescendo de tension psychologique.

Le plaisir spectatoriel se fonde sur l'incertitude découlant de l'impossibilité de prévoir si la conclusion de la série correspondra ou non au début du film Psycho. La correspondance (logique s'il s'agissait d'un prequel traditionnel) impliquerait un

37 Lefebvre, 1997, p. 23.

${ }^{8} 8$ Scahill, 2016.

39 Mittell, 2015. 
fonctionnement sur le mode du dispositif tragique, dans lequel les personnages ne peuvent pas échapper à leur destin. Ainsi, chaque meurtre ou accident concernant Norman et sa famille opère comme une possible actualisation de ce monde que nous connaissons; mais le récit, sériel, va constamment retarder l'arrivée de la confirmation finale. Néanmoins, les possibilités transfictionnelles sont ouvertes ${ }^{40}$, ce qui permet d'imaginer toutes sortes de déviation du tracé. Par exemple, dans les forums de discussion, certains téléspectateurs souhaitent que la série ne se termine pas et se poursuive au contraire jusqu'à vingt-cinq ans après les événements du film, ou encore que s'étire le temps entre le présent et le film Psycho.

Les métamorphoses et les agencements dépendant de l'acte de refaire sont, quant à eux, illimités. Ainsi, il est possible de remarquer que plusieurs éléments narratifs sont présentés de manière similaire, mais avec un rôle différent. On remarquera par exemple une réassignation des fonctions de victime et de bourreau dans la relation entre Norma et Norman, ou le retour, mais « relocalisé $4 \mathrm{I} »$, de certains noms, comme Emma (personnage de Psycho II et III). La série joue à recombiner les éléments : en les sélectionnant et, ensuite, en les recombinant, elle ne produit pas nécessairement la même «figure », mais fait émerger des résultats nouveaux. Le cas le plus intéressant est celui de l'image d'une femme blonde, criminelle mais gauche et malheureuse, qui, dans l'original, correspondait à Marion Crane et qui, dans Bates Motel, devient la mère de Norman. Norma Louisa Bates vit en effet une transformation significative par rapport à l'image que le spectateur se fait d'elle à partir des éléments les plus canonisés de la série culturelle ${ }^{42}$. Un commentateur le laisse entendre dans une remarque faite en ligne:

I am a bit confused, really. I suppose it is meant as a major reboot, in the sense that it is a completely alternate universe! In this, Norma Bates is NOT abusive to her son, in the least. In fact, she is a caring and compassionate woman who has had life totally handed to her in a flaming bag of horrible ${ }^{43}$.

Norma est un personnage à part entière, confronté à ses propres fantasmes; elle semble vouloir le bien de son fils, tentant de le protéger de lui-même. Dans un

$4^{\circ}$ Certaines séries jouent sur ces possibilités, comme Hannibal (NBC, 2013-2015), qui brouille les frontières entre backstory et expansion transfictionnelle.

${ }^{41}$ Francesco Casetti, The Lumierre Galaxy: Seven Key Words for the Cinema to Come, New York, Columbia University Press, coll. « Film and culture », 20I5.

${ }^{42}$ On mentionnera également l'existence d'une Elsa Bates dans le court métrage Mrs. Bates (Jara, 20IO).

43 Adrian, «Reviews \& Ratings for "Bates Motel” », IMDb, 25 mars 2016 http://www.imdb.com/user/ur29783597/?ref_=nm_bd_I (consultation le 2o septembre 2016). 
exemple frappant, on assiste à une « scène de la douche manquée » dans laquelle elle intervient pour sauver son propre fils et la jeune femme qu'il convoite ${ }^{44}$. Le protagoniste guette, se penchant à sa fenêtre, une cliente qui est dans sa propre chambre, mais il est violemment rappelé à l'ordre par sa mère avant qu'il ne puisse donner suite à ses désirs.

Norma semble en fin de compte être une mère aimante, consciente des troubles de son fils. Carlton Cuse, le créateur de la série, souligne l'importance du rapport d'«amour » entre mère et fils, une véritable réactivation d'éléments qui diverge du canon :

The story is ultimately 'how much can love conquer?' You have these two characters who their [sic] relationship is fatally flawed, and that's the essence of any tragedy. And yet we're hoping against hope that they can somehow stay together and keep what they have. That's the dramatic tension that's at the core of their relationship ${ }^{45}$.

Lorsque les personnages dorment dans le même lit, s'embrassent, se surveillent respectivement lors de leurs rencontres sentimentales, on reconnaît des échos de Psycho IV. Mais Norma est aussi une femme indépendante, qui tente de refaire sa vie en se consacrant à une activité professionnelle légitime. Cette connotation est renforcée par le paratexte traditionnel (bandes-annonces, affiches, photos promotionnelles, qui mettent en avant certains aspects de la série et jouent pour certains avec la référence au film d'Hitchcock) et par le paratexte transmédiatique. Les pages officielles des réseaux sociaux de la chaîne contribuent à construire une image rassurante de Norma. On remarque notamment un compte «Norma Bates » sur Pinterest qui correspond parfaitement à l'image léchée et ravissante que le personnage veut donner de lui-même : ses épingles sont des listes de recettes de gaufres, de belles photos d'ustensiles de cuisine, des détails des chambres du motel, ou encore des photos de famille montrant les personnages heureux dans leur quotidien, y compris dans leur passé avec le premier mari, dont on a gommé les caractéristiques centrales, soit le caractère violent et abusif (voir les figures 2 et 3 ).

Dans cette logique d'accrochage, l'extratexte joue également un rôle important. Comme jadis pour le remake de Van Sant, les personae des acteurs vont créer des frictions - qui d'ailleurs ne sont pas incompatibles avec une logique de transfiction - internes au monde. Elles appellent, grâce à l'encyclopédie

44 Bates Motel, saison 3, épisode I : «A Death in the Family », diffusé le 9 mars 2015.

45 Carlton Cuse, cité par Terri Schwartz, « Bates Motel takes Norman's creepy obsession with Norma to a new level », Screener, 20 avril 2015, http://screenertv.com/newsfeatures/bates-motel-norman-creepy-obsession-norma/ (consultation le 2o septembre 20I6). 
$1 \mathrm{~F}$

Norma Bates
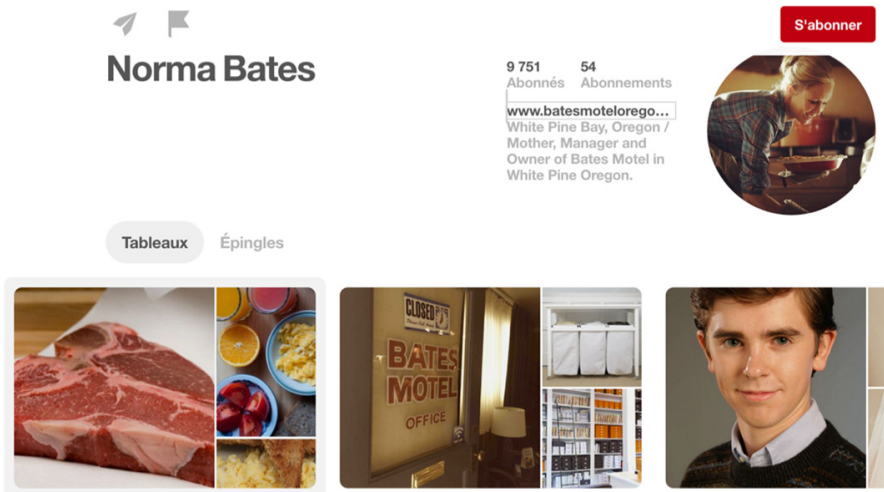

// Home Cooking //

S'abonner

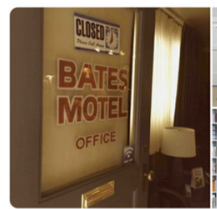

// Bates Motel Déc... 233 Épingles

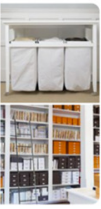

S'abonner

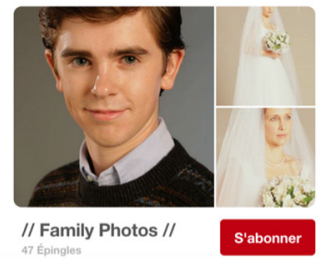

Fig. 2. « Family photos », Compte Pinterest de Norma Bates, https://www.pinterest.com /NormaBatesMotel/family-photos/ (consultation le I9 avril 2017).

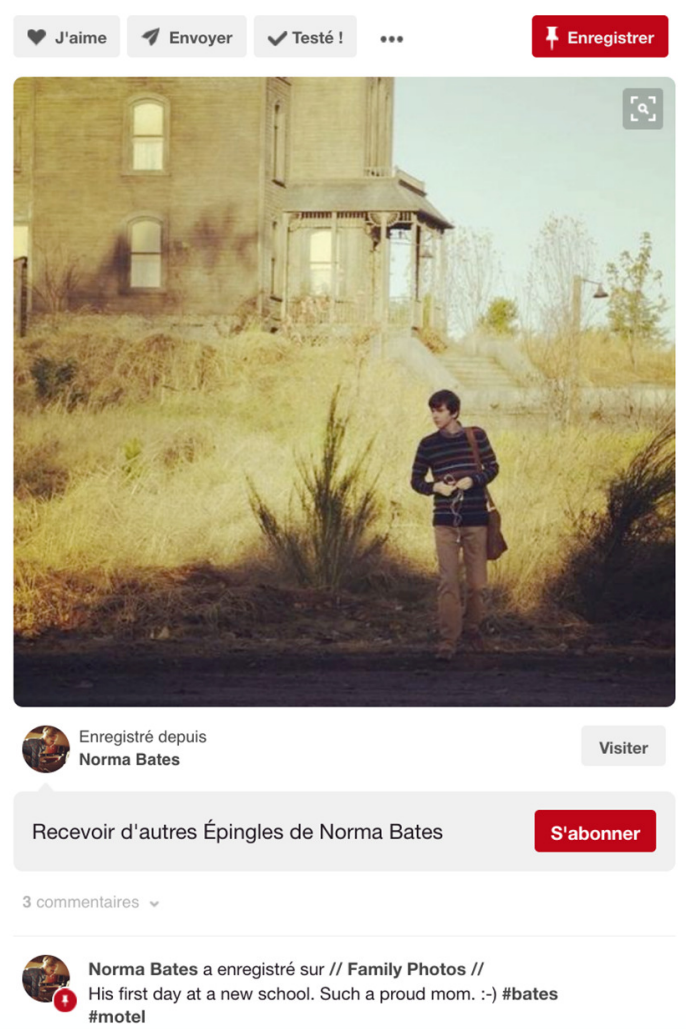

Fig. 3. « Le premier jour d'école de Norman», Compte Pinterest de Norma Bates, https://www.pinterest.com/pin/563724078327516I8I/ (consultation le 19 avril 2017). 
intertextuelle qui s'inscrit dans leurs corps, de nouveaux publics et permettent de relire - donc de refaire - le texte originaire. Pour Bates Motel, si Vera Farmiga (Norma), Max Thieriot (Dylan) et Olivia Cooke (Emma Decody) ont joué dans des films d'horreur, c'est le comédien qui joue Norman qui apporte une touche très particulière à l'ensemble. Freddie Highmore jouait en effet dans Finding Neverland (Marc Forster, 2004), où il incarnait un enfant ne voulant pas grandir qui a inspiré J. M. Barrie dans l'écriture de Peter Pan. Un effet intéressant se produit dans Bates Motel par la friction avec ce monde possible. Considérons le dialogue suivant :

Emma: I love your mom, but I think she loves you so much that she can't stand the thought of you growing up. So maybe this gives you a feeling that being an adult, being sexual, is somehow wrong.

Norman: Well, she does the best she can. We're just close.

Emma: Yeah, and you always will be, but that doesn't mean you can't grow up. Unless you're Peter Pan. Do you want to be Peter Pan?

Norman: Maybe. If you'll be Wendy.

Emma: Peter and Wendy never got to have $\operatorname{sex}^{46}$.

Peter Pan, l'enfant qui refuse de grandir, est une autre série culturelle qui parcourt l'ensemble du système Psycho-Bates Motel en la reliant à d'autres textes et qui permet de souligner, sous un autre jour, la relation trouble de Norman avec sa mère.

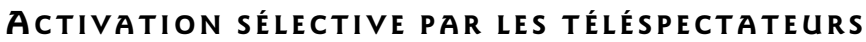

Les productions discursives des téléspectateurs peuvent également donner vie à des réactivations sélectives, dans un sens canonique par rapport à Bates Motel et non canonique par rapport au film de 1960, ou même non canonique par rapport à Bates Motel. Rappelons que pour certains la série représente le seul texte de référence. Un débat anime d'ailleurs les pages de Reddit :

Usager ceazy905

Je n'ai jamais vu le film Psycho et je ne connais rien à ce sujet

\section{Usager McIgglyTuffMuffin}

J'ai la sensation que tout ce qui entoure Psycho est tellement enraciné dans la culture populaire que ton cerveau fait automatiquement la connexion.

${ }^{46}$ Bates Motel, saison 3, épisode 2 : « The Arcanum Club », diffusé le I6 mars 20I5. 
Seulement à écouter Bates Motel, pour moi en tout cas, ma pensée va immédiatement à Psycho à cause de l'association des noms ${ }^{47}$.

La relation entre Norma Bates et le shérif Alex Romero, personnage qui revêt un rôle de plus en plus important au fil des saisons, est au centre de plusieurs fan fictions. Le nombre de textes consacrés au couple « Normero » (de la crase de ces deux noms) prouve l'importance et la réussite de l'activation sélective du personnage de Norma dans la série télé. Les fan fictions proposent aussi des options non canoniques aux actions représentées dans le texte télévisuel. Par exemple, l'épisode final de la saison quatre, qui contient un coup de théâtre d'importance capitale, est réécrit plusieurs fois par les fans, qui (de manière non canonique par rapport au film de 1960) ne sont pas prêts à accepter la conclusion officielle. Et lorsque le passé de Norma et de ses enfants est exploré, ces réécritures montrent que la série devient un espace de discours dilatable, explorable, au sein de l'espace de discours plus large qu'est le monde des Bates.

Si on observe d'autres productions, notamment les parodies sur YouTube, Norman Bates se rapproche d'un signifiant flottant, à mobiliser avec un investissement minimal de ressources, évoquant rapidement une séquence culte, le meurtre sous la douche, et jouant sur la phrase-slogan de la série d'A\&E, «A boy's best friend is his mother ${ }^{48} \gg$. Les parodies renforcent l'étrangeté de la superposition des deux identités Norman/mère, en exploitant pleinement le potentiel de travestissement burlesque du film (déjà vu au moins dans High Anxiety, Mel Brooks, 1977). Soulignons également les pages Pinterest consacrées à la série, qui relèvent proprement d'une logique d'《 accrochage ».

Lorsqu'on analyse les traces des discours et des usages des fans concernant ce phénomène, on remarque aussi des résultats émergents qui ne touchent pas à la composition logique du récit, des lectures que Janet Staiger appellerait perverses ${ }^{49}$ et qui font surgir d'autres liens et des interférences affectives. Par exemple, un téléspectateur attribue du sens au retour d'un même nom, brouillant les frontières entre le monde de la production et le monde de la fiction : Vera Miles, actrice de Psycho, et Vera Farmiga, actrice de Bates Motel. Il s'agit dans ce cas d'un usage

47 Ceazy905 et McIgglyTuffMuffin, «Anyone else loves Bates Motel but never seen Psycho the original or remake/s ? », Reddit, www.reddit.com/r/BatesMotel /comments/4h84r8/anyone_else_loves_bates_motel_but_never_seen/ (consultation le is septembre 2016).

$4^{8}$ How I Am Your Mother (Psycho / How I Met Your Mother / Bates Motel Parody), And You Films, 2013, Youtube: https://www.youtube.com/watch?v=ZBYvxL44KUY (consultation le is septembre 2016).

49 Janet Staiger, Perverse Spectators: The Practices of Film Reception, New York, Londres, New York University Press, 2000. 
dépourvu de toute valeur heuristique dans le cadre d'une interprétation scientifique. Toutefois, pour certains fans, il représente l'activation d'un point de contact significatif, capable de renforcer l'inscription de la série dans la série culturelle de Psycho. L'analyse de la réception représente donc un complément important dans l'étude de ce reenactment qu'est Bates Motel: elle montre la possibilité d'explorer à souhait le monde complexe que la série d'A\&E active et de remarquer d'ultérieures réactivations sélectives de portions de contenu.

\section{CONCLUSIONS}

La sphère de discours de Psycho est composée de plusieurs logiques d'accrochage, souvent contradictoires les unes avec les autres, qui pourtant ne nuisent pas à l'existence de chacune d'entre elles. La mise en série est ainsi génératrice de systèmes complexes, d'agencements d'éléments en circulation, pouvant produire, par leur rapprochement, des effets imprévisibles sur le plan sémiotique comme sur le plan formel, qui négocient et, de surcroît, réaffirment constamment l'identité du système.

Bien que l'on puisse affirmer, avec Roy Menarini, qu'Hitchcock est le « lieu citable par excellence ${ }^{5} \gg$ de l'histoire du cinéma de l'après-guerre, devenant un véritable genre, avec ses modes de mise en scène et ses invariants syntaxiques, et que Leitch parlait au sujet de Gus Van Sant du «simulacre d'un certain Hitchcock présentement à la mode ${ }^{{ }^{\mathrm{I}}} \gg$, Bates Motel ne cherche pas à refaire du Hitchcock. La série s'affranchit de toute anxiety of influence, n'allant pas non plus renforcer une auctorialité autre qui remplacerait celle d'Hitchcock — ou qui se lirait en tension avec la primauté historique de celle-ci. Bates Motel est l'appropriation exploratoire d'un monde. L'enjeu ne se trouve pas dans la définition d'un auteur comme pouvait l'être Gus Van Sant, en tant que nouvel exécuteur de l'œuvre, évoquant ainsi la dimension de l'héritage ${ }^{2}$, mais demeure dans les effets créés par la superposition de résultats qui apparaissent dans l'espace sériel.

À une époque marquée par le fonctionnement en réseau, le musée imaginaire se présente comme une stratégie nécessaire pour la réception comme pour la création, devant composer avec des éléments inscrits dans un imaginaire commun, des textes cultes, et une productivité participative des téléspectateurs. Le concept d'activation

so Roy Menarini, La strana coppia. Studi sullintertestualità e la parodia del cinema, Pasian di Prato (Udine), Campanotto, coll. «Zeta cinema », 2004, p. II2.

5I Thomas Leitch, « Hitchcock without Hitchcock », Literature/Film Quarterly, vol. 3I, $\mathrm{n}^{\circ} 4,2003$, p. 257.

52 Chambat-Houillon, 2005, p.29. 
ou de réactivation sélective, dans le cadre de la série culturelle et du musée imaginaire, nous a permis de donner un sens aux métamorphoses d'un univers culte à travers quelques-unes de ses récentes incarnations médiatiques. L'acte de refaire semble dès lors une nécessité pour les créateurs comme pour les spectateurs, dans l'espace intermédiatique marqué par une circulation des fragments : il est le garant de la vitalité et de l'expansion de sphères de discours, dans une logique sérielle en réseau. 


\title{
Psycho/Bates Motel : hyperdiégèse et réactivation sélective
}

\author{
Martha Boni, Université de Montréal
}

\section{RÉSUMÉ}

Cette contribution propose un modèle pour l'analyse de la tendance contemporaine consistant à refaire, en série, un film culte. L'analyse de la série Bates Motel (A\&E, 2013-), choisie comme exemple pertinent, s'appuie sur l'insertion de la série dans une sphère de discours plus large représentée par l'univers de Psycho et ses multiples incarnations médiatiques, incluant les appropriations «apocryphes » des téléspectateurs. Bates Motel correspond ainsi à l'exploration d'un espace. Ce phénomène est décrit à travers les concepts d'hyperdiégèse de Matt Hills (Fan Cultures, Londres, New York, Routledge, 2002), ainsi que de série culturelle et de musée imaginaire de Martin Lefebvre (Psycho: de la figure au musée imaginaire. Théorie et pratique de l'acte de spectature, Montréal, Paris, L'Harmattan, 1997). Les notions d' « activation » ou de « réactivation sélective » sont par ailleurs proposées et discutées.

\section{ABSTRACT}

This paper proposes a model for the analysis of the contemporary trend of redoing a cult movie as a television series. The analysis of the show Bates Motel (A\&E, 2013-), chosen here as a primary example, is grounded in an understanding of the series as a singular sphere integrated within the much wider sphere of discourse initiated by the original film Psycho (1960), including its multiple media incarnations and apocryphal fan-made works. Bates Motel therefore incorporates an exploration of this media sphere. The paper traces this phenomenon through Matt Hills' concept of hyperdiegesis (Fan Cultures, Londres, Routledge, 2002), and Martin Lefebvre's concepts of cultural series and the imaginary museum (Psycho: de la figure au musée imaginaire. Théorie et pratique de l'acte de spectature, Montréal, Paris, L'Harmattan, 1997). The notions of "selective activation" and "reactivation" are also put forward and discussed.

\section{NOTE BIOGRAPHIQUE}

MARTA BoNI est professeure adjointe au Département d'histoire de l'art et d'études cinématographiques de l'Université de Montréal. Elle s'intéresse aux problèmes théoriques et méthodologiques liés aux phénomènes de type sériel et à leurs mutations dans le panorama contemporain des cultures numériques: séries télé, 
adaptation, transfiction, transmédia, world building. Elle explore la complexité narrative de ces phénomènes et l'entrelacement de celle-ci avec les pratiques de la réception. Elle mène actuellement le projet de recherche « Les séries télé à l'ère de la culture numérique. Production, diffusion, réception et circulation en réseau ». 